\title{
Retrograde nailing for distal femur fractures in the elderly
}

\author{
Jasdeep Giddie*, Seif Sawalha, and Martyn Parker \\ Department of Orthopaedics, Peterborough and Stamford Hospital NHS Foundation Trust, Peterborough City Hospital, Bretton Gate, \\ PE3 9GZ Peterborough, UK
}

Received 10 July 2015, Accepted 2 November 2015, Published online 1 December 2015

\begin{abstract}
Introduction: We report the results of treating a series of 56 fractures in 54 elderly patients with a distal femur fracture with a retrograde femoral nail.

Methods: Fifty-four of the nails were inserted percutaneously with a closed reduction. After surgery all patients were allowed to weight bear as tolerated. Four fractures were supported in a temporary external splint.

Results: The mean age of patients was 80.6 years (range 51-103 years), 52/54 (96\%) were females. There were no cases of nail related complications and no re-operations were required. One patient was lost to follow up. The 30-day mortality was 5/54 (9.3\%) and the one year mortality was $17 / 54(31.5 \%)$.

Conclusions: Distal femoral nail fixation provides a good method of fixation allowing immediate mobilisation for this group of patients.
\end{abstract}

Key words: Distal femur fracture, Retrograde nail, Union, Complications, Mortality.

\section{Introduction}

Distal femur fractures are relatively rare injuries accounting for approximately $1 \%$ of fractures in the elderly [1]. They have bimodal age distribution; young patients as a result of high-energy injuries and elderly patients after simple falls [2]. Despite affecting the same anatomical location as young patients, fractures in the elderly pose different challenges due to osteoporotic bone and the overall patients' medical condition [3]. The aim of surgical management in these frail patients should be considered similar to those with a proximal femur fracture, that is a quick surgical procedure that allows early weight bearing and mobilisation to avoid the complications of prolonged bed rest.

Retrograde nailing is an established management option for these fractures. Previous studies on the outcome of patients managed by retrograde nailing often included both young patients with high-energy fractures and elderly patients with osteoporotic fractures [4-6]. However, the differences in patient and fracture characteristics between these two groups make them two distinct injuries requiring a separate analysis.

We aimed to establish, that the principles of management of osteoporotic distal femoral fractures should mimic the treatment of proximal femoral fractures, i.e. minimally invasive, early surgery with a stable construct to allow unrestricted weight bearing.
We present one of the largest case series of osteoporotic distal femoral fractures managed by the senior author (MJP), a consultant, with a specialist interest in fragility fractures.

\section{Patients and methods}

The inclusion criteria were all patients admitted to Peterborough City Hospital between 1999 and 2014 with a distal femoral fracture treated with a retrograde femoral nail. Any fractures treated with an alternative fixation device and fractures managed non-operatively were excluded from the study. Data was collected using a standard proforma started at the time of the patients' admission and included documentation of residential status, ASA grade [7] and mobility score. Fractures were classified using the AO system [8]. The main outcomes studied were mortality and the occurrence of fracture healing complications. After discharge from hospital patients were followed up in a specific fracture clinic. One-year mortality rates were determined using local patient register data for the patients.

There were a total of 56 fractures in 54 patients during the study period. Two patients presented with bilateral distal femur fractures. Associated injures were present in two patients. These were an ipsilateral shoulder fracture and ipsilateral ankle. Four patients had a knee arthroplasty in place, three a sliding hip screw in place, two a proximal intramedullary nail and one a hip arthroplasty (Table 1).

\footnotetext{
*Corresponding author: jgiddie@gmail.com
} 
Table 1. Patient demographics.

\begin{tabular}{lc}
\hline Patient numbers & 54 \\
Number of fractures & 56 \\
Mean age (range) & $80.6(51-103$ years) \\
Female (\%) & $53(98.2 \%)$ \\
From own home (\%) & $40(74.1 \%)$ \\
Residential home (\%) & $5(9.3 \%)$ \\
Nursing home (\%) & $8(14.8 \%)$ \\
Fall in hospital (\%) & $2(3.7 \%)$ \\
Pathological fractures (\%) & $4(7.4 \%)$ \\
Mean haemoglobin g/L & 11.7 \\
Mean ASA grade & 2.7 \\
No walking aids (\%) & $19(35.2 \%)$ \\
One/two sticks (\%) & $9(16.2 \%)$ \\
Walking frame (\%) & $18(33.3 \%)$ \\
Immobile (\%) & $8(14.8 \%)$ \\
AO classification & Number of fractures \\
33 A - 1 & 39 \\
33 A - 2 & 13 \\
33 A - 3 & 4 \\
\hline
\end{tabular}

For the eight immobile patients, the outcome of surgical management differed, with the emphasis being to allow essential nursing care to be performed without discomfort to the patients.

Surgery was undertaken after informed consent was obtained. The patients were placed supine on a radiolucent fracture table. The knee was placed in 20-30 degrees of flexion with a radiolucent supporting pad placed behind the distal femur. We used image guidance and a radio opaque ruler placed on the patient's thigh over the fracture site to determine the length and diameter of the nail. Where possible the fracture was reduced closed. A 2-3 cm longitudinal incision was made immediately medial to the patellar tendon and access to the knee either medial to the tendon or using a tendon splitting approach. A guide wire was introduced manually through the intercondylar notch, above the insertion of the posterior cruciate ligament along the distal femur and its position checked under image guidance on two planes. A $12.5 \mathrm{~mm}$ hollow reamer was inserted over the guide wire. The selected nail was attached to the targeting device and introduced under image guidance. Distal fixation used the targeting device via stab incisions. For nails greater than $300 \mathrm{~mm}$, proximal locking is achieved free hand from an anterior to posterior direction. For the long nails the aim was to place the tip of the nail in trochanteric region.

A Targon retrograde nail (RF) for 52 fractures (Figure 1) and a Zimmer retrograde nail for four cases were used. Open reduction of the fracture was required in two fractures, with the remainder being reduced closed. Two distal locking screws were used in two fractures, three distal screws for 11 fractures and four screws for the remainder. Proximal locking with one screw was undertaken for nine fractures and with two screws for 43 fractures. Twelve of the nails were short (length 160 $240 \mathrm{~mm}$ ) and all of them had proximal screws inserted using the alignment jig. The remainder were long nails (range $300 \mathrm{~mm}-400 \mathrm{~mm}$ ). For those patients with a knee prosthesis in place the nail was passed between the condyles. For those with a sliding hip screw in place the nail was passed past the distal part of the plate and any screws that impeded passage of the nail were removed percutaneously. The two patients with a proximal femoral nail had this nail removed at the start of surgery.

Surgery was undertaken or directly supervised by the senior author (MJP) for 52 patients. Post-operative management of all the patients was undertaken by the senior author (MJP). For four patients an external splint was used to support the fracture for a period of six weeks. All these fractures were close to the knee joint with limited support from the distal locking screws. All patients were encouraged to mobilise as able with aids and weight bear as tolerated. After discharge from hospital patients were followed up in a specific fracture clinic.

\section{Results}

The mean number of hours to surgery was 38 (range 7$137 \mathrm{~h}$ ). Mean operative time was $82 \mathrm{~min}$ and mean operative blood loss $205 \mathrm{~mL}$. After surgery 17 patients required a blood transfusion. All patients were allowed to weight bear as able. For four patients an external splint was used to support the fracture for a period of six weeks. All these fractures were close to the knee joint with limited support from the distal locking screws. The median hospital stay was 14 days (mean 22.7 days, range 3-76). This included the time spent on any additional rehabilitation wards till the patient was discharged home.

All superficial wound infections were treated successfully with a course of antibiotics (Table 2).

Nail-related complications included one prominent distal screw. This was removed under local anaesthetic as a day case procedure.

Post-operative follow-up was conducted until clinical and radiological union occurred. Mean clinical and radiographic follow-up was 123 days (range 38-365 days). For one patient the fracture line was still visible at one year but no treatment was required. All the other fractures healed uneventfully and no other patient required an additional surgery for the fracture.

Mortality for up to one year from injury is shown in Figure 2. One patient was lost to follow-up at 63 days from injury.

\section{Discussion}

This is a series of elderly patients with distal femur fractures treated with a retrograde femoral nail. Other than the cases of sepsis there were no implant-related complications. This is despite the policy of early mobilisation and the allowing of weight bearing as able. In addition, the surgery was as minimally invasive as possible. This means this type of operative procedure is suitable for frail elderly. We have applied the same principles to this group of patients as we use for the elderly hip fracture patients, that is early technically correct surgery minimally invasive surgery followed by immediate and unrestricted mobilisation.

We did not encounter any implant-related complications in our study. Other researchers reported some complications including femoral fractures above the nail, fracture of the implant, nail protrusion through the knee and fixation failure 
(a)
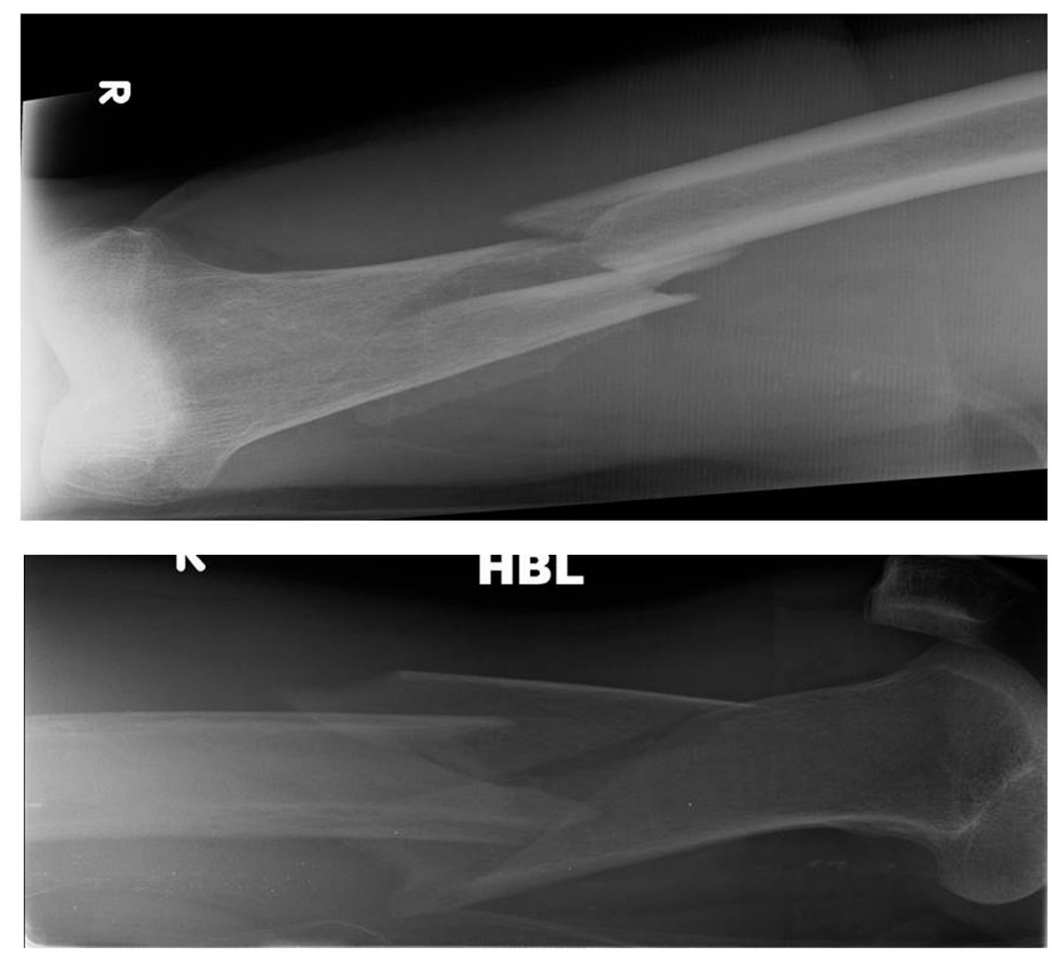

(b)
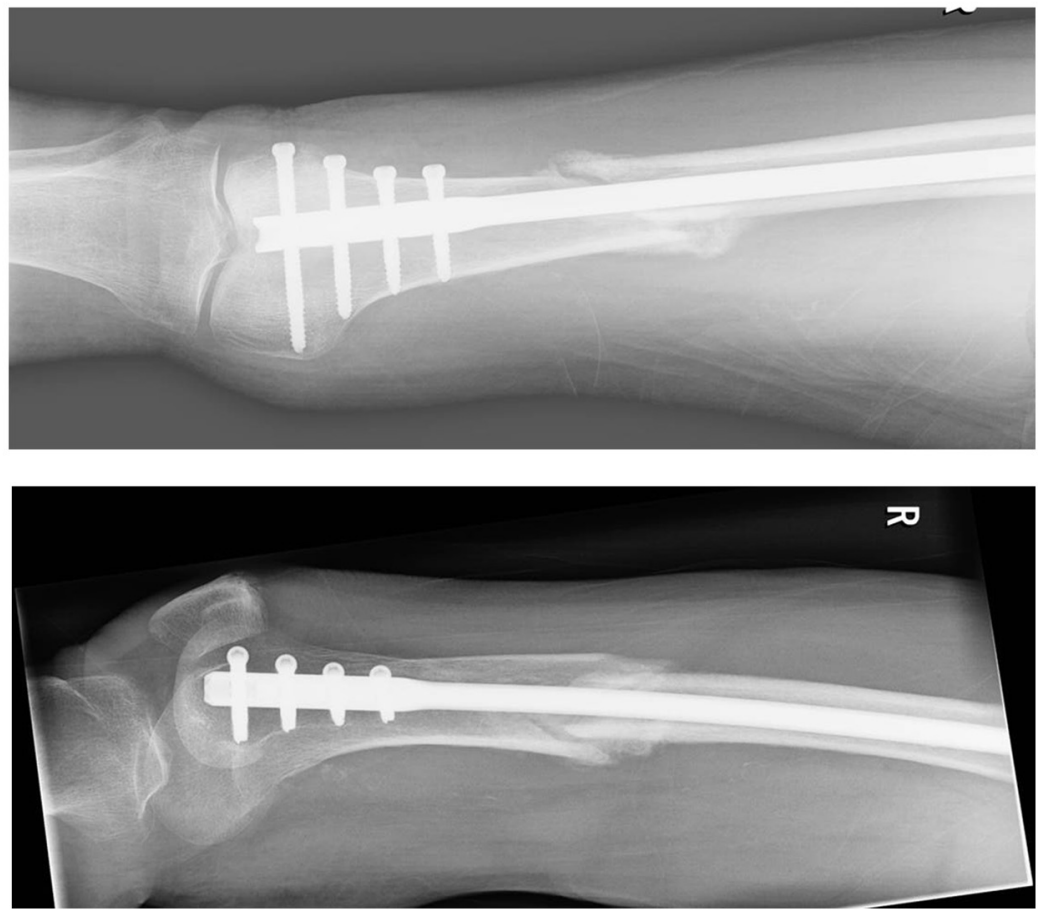

Figure 1. (a) Pre- and (b) post-operative radiographs of distal femur fracture treated with Targon RF nail.

requiring revision surgery [9-12]. Overall, the mortality rate in distal femur fracture patients was higher in our study than those previous reports [13]. The mean age of patients in our study was higher than those of other studies, which could explain the higher mortality rate.

In this series of elderly patients with osteoporotic distal femoral fractures, we included a 51-year-old male patient. Although this is not considered elderly, the patient was in a nursing home, immobile and sustained a low-energy injury. Given the circumstances through which the fracture occurred he has been included in this study.

The union rate in our study is comparable to published results using other intramedullary devices in elderly patients with distal femur fractures after low-energy trauma. Moskal and O'Shea reported two non-unions in their case series of 14 fractures and Kumar et al. reported one non-union in their 
Table 2. Post-operative complications.

\begin{tabular}{ll}
\hline Superficial wound infection & 4 \\
Pneumonia & 3 \\
Fat embolism & 2 \\
Deep vein thrombosis & 1 \\
Post-operative delirium & 1 \\
\hline
\end{tabular}

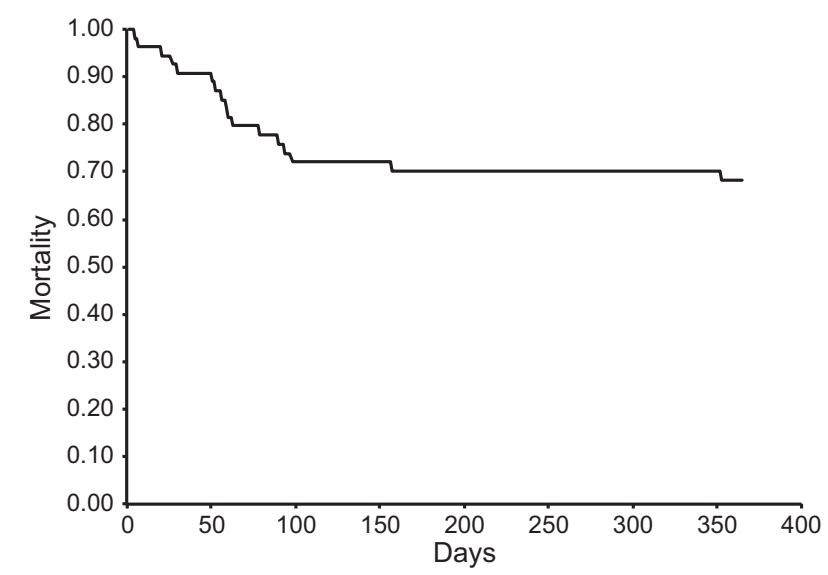

Figure 2. Kaplan Meier one-year survival curve for distal femoral fractures.

case series of 18 distal femur fractures [9, 10]. El-Kawy et al. reported no non-unions in their study of 23 fractures [14]. A significant proportion of our patients died before union could be assessed which reflects the fragility of this population of patients.

There are several limitations to this study. We did not have data on the range of knee movement or functional outcome measures such as post-operative mobility scores. However, this is a frail group of patients who often have a degree of cognitive impairment limiting availability of such data. This is also reflected in the relatively high proportion of patients who died before fractures were followed up to fracture union. A further limitation of this study is that two different types of retrograde nails were used although the design of these nails was very similar.

This paper has three key messages: In elderly patients with an extra-articular distal femoral fracture, surgical treatment using a retrograde femoral nail is reliable. A minimally invasive approach with unrestricted mobilisation has few nailrelated complications. Thirdly, despite early intervention there remains a high rate of mortality.

As the numbers of distal femoral fractures continue to increase, future research in this field should continue to develop to treat more complex fracture patterns, using a minimally invasive approach, permit early weight bearing and aim to reduce the significantly high mortality rate.

In conclusion, we have shown satisfactory results using a retrograde femoral nail in the management of elderly patients with a distal femoral fracture. Early and unrestricted mobilisation is possible with a very low risk of fracture healing complications.

\section{Conflict of interest}

JG, SS and MP declare no conflict of interest in relation with this paper.

\section{References}

1. Court-Brown CM, Clement ND, Duckworth AD, Aitken S, Biant LC, McQueen MM (2014) The spectrum of fractures in the elderly. Bone and Joint Journal 96-B, 366-372.

2. Martinet O, Cordey J, Harder Y, Maier A, Buhler M, Barraud GE (2003) The epidemiology of fractures of the distal femur. Injury 31(suppl 3), C62-C63.

3. Wade PA, Okinaka AJ (1959) The problem of the supracondylar fracture of the femur in the aged person. Am J Surg 97, 499-512.

4. Seifert J, Stengel D, Matthes G, Hinz P, Ekkernkamp A, Ostermann PA (2003) Retrograde fixation of distal femoral fractures: results using a new nail system. J Orthop Trauma 17, 488-495.

5. Garnavos C, Lygdas P, Lasanianos NG (2012) Retrograde nailing and compression bolts in the treatment of type $\mathrm{C}$ distal femoral fractures. Injury 43, 1170-1175.

6. Ghandour A, Cosker TD, Kadambande SS, Pallister I, Russell ID (2006) Experience of the T2 supracondylar nail in distal femoral fractures. Injury 37, 1019-1025.

7. American Society of Anaesthesiologists (1963) New classification of physical status. Anaesthesiology 24, 111.

8. Muller ME, Nazarian S, Koch P, Schatzker J (1990) The AO classification of fracture of long bones. Berlin, Heidelberg, New York, London, Paris, Tokyo, Hong Kong, Springer-Verlag.

9. Moskal JT, O'Shea JJ (2003) Intramedullary stabilization of distal-third femur fractures in octogenarians. J South Orthop Assoc 12, 3-8.

10. Kumar A, Jasani V, Butt MS (2000) Management of distal femoral fractures in elderly patients using retrograde titanium supracondylar nails. Injury 31, 169-173.

11. Dunlop DG, Brenkel IJ (1999) The supracondylar intramedullary nail in elderly patients with distal femoral fractures. Injury 30, 475-484.

12. Moore TJ, Watson T, Green SA, Garland DE, Chandler RW (1987) Complications of surgically treated supracondylar fractures of the femur. J Trauma 27, 402-406.

13. Streubel PN, Ricci WM, Wong A, Gardner MJ (2011) Mortality after distal femur fractures in elderly patients. Clin Orthop Relat Res 469, 1188-1196.

14. El-Kawy S, Ansara S, Moftah A, Shalaby H, Varughese V (2007) Retrograde femoral nailing in elderly patients with supracondylar fracture femur; is it the answer for a clinical problem? Int Orthop 31, 83-86.

Cite this article as: Giddie J, Sawalha S \& Parker M (2015) Retrograde nailing for distal femur fractures in the elderly. SICOT J, 1, 31 